\title{
Development of glaucoma predictive model and risk factors assessment based on supervised models
}

Mahyar Sharifi ${ }^{1}$, Toktam Khatibi ${ }^{1 *}$, Mohammad Hassan Emamian ${ }^{2}$, Somayeh Sadat ${ }^{3}$, Hassan Hashemi ${ }^{4}$ and Akbar Fotouhi ${ }^{5}$

\author{
*Correspondence: toktam.khatibi@ \\ modares.ac.ir \\ ${ }^{1}$ School of Industrial and Systems \\ Engineering, Tarbiat Modares \\ University, Tehran, Iran \\ Full list of author information is \\ available at the end of the article
}

\begin{abstract}
Objectives: To develop and to propose a machine learning model for predicting glaucoma and identifying its risk factors.

Method: Data analysis pipeline is designed for this study based on Cross-Industry Standard Process for Data Mining (CRISP-DM) methodology. The main steps of the pipeline include data sampling, preprocessing, classification and evaluation and validation. Data sampling for providing the training dataset was performed with balanced sampling based on over-sampling and under-sampling methods. Data preprocessing steps were missing value imputation and normalization. For classification step, several machine learning models were designed for predicting glaucoma including Decision Trees (DTs), K-Nearest Neighbors (K-NN), Support Vector Machines (SVM), Random Forests (RFs), Extra Trees (ETs) and Bagging Ensemble methods. Moreover, in the classification step, a novel stacking ensemble model is designed and proposed using the superior classifiers.

Results: The data were from Shahroud Eye Cohort Study including demographic and ophthalmology data for 5190 participants aged 40-64 living in Shahroud, northeast Iran. The main variables considered in this dataset were 67 demographics, ophthalmologic, optometric, perimetry, and biometry features for 4561 people, including 4474 non-glaucoma participants and 87 glaucoma patients. Experimental results show that DTs and RFs trained based on under-sampling of the training dataset have superior performance for predicting glaucoma than the compared single classifiers and bagging ensemble methods with the average accuracy of 87.61 and 88.87 , the sensitivity of 73.80 and 72.35 , specificity of 87.88 and 89.10 and area under the curve (AUC) of 91.04 and 94.53 , respectively. The proposed stacking ensemble has an average accuracy of 83.56 , a sensitivity of 82.21 , a specificity of 81.32, and an AUC of 88.54.
\end{abstract}

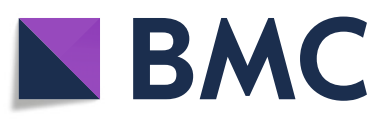

(c) The Author(s). 2021 Open Access This article is licensed under a Creative Commons Attribution 4.0 International License, which permits use, sharing, adaptation, distribution and reproduction in any medium or format, as long as you give appropriate credit to the original author(s) and the source, provide a link to the Creative Commons licence, and indicate if changes were made. The images or other third party material in this article are included in the article's Creative Commons licence, unless indicated otherwise in a credit line to the material. If material is not included in the article's Creative Commons licence and your intended use is not permitted by statutory regulation or exceeds the permitted use, you will need to obtain permission directly from the copyright holder. To view a copy of this licence, visit http://creativecommons.org/licenses/by/4.0/. The Creative Commons Public Domain Dedication waiver (http://creativecommons.org/publicdomain/zero/1.0/) applies to the data made available in this article, unless otherwise stated in a credit line to the data. 
Conclusions: In this study, a machine learning model is proposed and developed to predict glaucoma disease among persons aged 40-64. Top predictors in this study considered features for discriminating and predicting non-glaucoma persons from glaucoma patients include the number of the visual field detect on perimetry, vertical cup to disk ratio, white to white diameter, systolic blood pressure, pupil barycenter on $Y$ coordinate, age, and axial length.

Keywords: Ophthalmology, Data Mining, Imbalanced Learning, Feature selection, Ensemble classification

\section{Introduction}

Glaucoma is the second cause of irreversible blindness and the fourth cause of Moderate and Severe Vision Impairment (MSVI) in the world.[1] Glaucoma prevalence rises with age increasing, and it is one of the main risk factors for blindness and MSVI in people older than 50.[1].

The average number of persons who go blind from glaucoma has been increased from 2.5 million persons in 1990 to 3 million persons in 2015. Moreover, the average number of persons and the average number of persons who suffer from MSVI due to glaucoma has been raised from 3 million persons to 4 million persons from 1990 till 2015.[2].

It has been predicted that the number of persons suffering from glaucoma aged between 40 and 80 years old would be increased from 64.3 million persons in 2013 to 76 million persons in 2020 and 111.8 million persons in 2040.[3].

Previous studies have identified different risk factors such as age, gender, race, Interocular Pressure (IOP), diabetes, and family history for glaucoma.[4-7].

Among different studies performed in the population older than 40, the glaucoma prevalence has been reported from 1.44 to $4 \%$. In contrast, more than $50 \%$ of patients suffering from glaucoma have been unaware of their illness.[5, 8, 9] The identified risk factors for glaucoma in Iran are age, IOP, diabetes, axial length, and gender.[5].

Glaucoma is usually an asymptomatic disorder. If a severe visual impairment is not detected, the patient will not be aware of their disease. Therefore, some physicians have called glaucoma the silent thief of sight.[10].

The blindness occurring due to glaucoma is irreversible, but some early treatment such as reducing IOP and some surgical interventions can cease blindness due to glaucoma. However, some early treatment activities such as reducing IOP and some surgical operations can help control the disease progression. Early detection and diagnosis of glaucoma and identifying high-risk groups can reduce the irrecoverable adverse effects of glaucoma.

Artificial intelligence and machine learning methods have various applications in solving medical and healthcare problems[11-13], such as in ophthalmology[14]. Developing automatic methods for ophthalmological diseases[15], ophthalmologic image analysis[16], network analysis for gene expression data for eye diseases[17], predicting the progressions of ophthalmologic diseases[18], and evaluating eye diseases' progression[19-21] are some of these applications.

The automatic methods for predicting and diagnosing glaucoma can be used as the computer-assisted diagnosis (CAD) method and decision support system (DSS) to 
improve glaucoma diagnosis and management accuracy. In this study, the main aim is to design and develop machine learning models for glaucoma prediction. For this purpose, demographic characteristics, optometry, biometry, perimetry features, and ophthalmologic examination results are used as the input variables.

The main novelty of this study lies in several folds, including:

- Proposing and designing a two-step classification task: The first step includes training the base and single classifiers on the training dataset and evaluating their performance based on a subset of the training dataset named as validation dataset, finding the superior classifiers. The second step is designing a novel stacked ensemble classifiers based on the superior classifiers.

- Using comprehensive ophthalmology features like perimetry and biometry to develop the glaucoma prediction model without any fundus features. Features of this study come from non-interventional ophthalmologic examinations.

- To address a highly imbalanced dataset with 87 instances in the glaucoma class (1.9\% of all instances) without generating artificial instances.

- Analysing a cohort dataset.

\section{Literature review}

Different methods based on artificial intelligence and machine learning have been used in various applications for glaucoma management in recent years. [22] For instance, building glaucoma interaction networks [17], assessing the optic disk [23, 24], and detecting visual field progression[19-21], diagnosis, and screening.[10, 11, 25-33].

To propose and develop models to screen and diagnose diseases using structured datasets and complex data such as medical images and gene expression data can assist the physicians in early managing and diagnosing glaucoma. [10, 11, 31, 32]

Table 1 summarizes the related works considering model development for screening and/or diagnosis of glaucoma.

As listed in Table 1, several previous studies have proposed models for diagnosing and screening glaucoma using different features and datasets. The mentioned models can be used to assist physicians in early diagnosis and decision-making. Fundus images in most previous studies were used as the vital input data for developing glaucoma diagnosing and screening models. In a few previous studies, genome data were used to improve glaucoma diagnosing and screening models because genetic and race are two common risk factors for glaucoma identified in previous researches. Although fundus images and genome data have an excellent performance in diagnosing glaucoma, these data types increase the costs and complexity of models. These two data types with some structured data from other ophthalmologic examinations and clinical data can improve the performance of models.

This study used different features, including demographic characteristics, optometry, biometry, perimetry, and ophthalmologic examination results, to help glaucoma prediction.

\section{Materials and methods}

In this study, the 'Shahroud eye cohort study' dataset [34] is analyzed for predicting glaucoma based on Cross-Industry Standard Process for Data Mining (CRISP-DM) [35] 
Table 1 Summary of the previous studies considering glaucoma prediction, screening and/or diagnosis

\begin{tabular}{|c|c|c|c|c|c|}
\hline \multirow[t]{2}{*}{ Authors } & \multicolumn{2}{|l|}{ Target } & \multirow{2}{*}{$\begin{array}{l}\text { Type of } \\
\text { Dataset }\end{array}$} & \multirow[t]{2}{*}{ Dataset } & \multirow[t]{2}{*}{ Classifier } \\
\hline & Diagnosis & $\begin{array}{l}\text { Do } \\
\text { Screening } \\
\text { or Not }\end{array}$ & & & \\
\hline $\begin{array}{l}\text { (Li et al., } \\
\text { 2019)[31] }\end{array}$ & Yes & Yes & Structured & SAP Data & $\begin{array}{l}\text { LDA, SVM, NB, } \\
\text { ANN }\end{array}$ \\
\hline $\begin{array}{l}\text { (Liu et al., } \\
\text { 2013)[10] }\end{array}$ & Yes & Yes & $\begin{array}{l}\text { Structured, } \\
\text { Images and } \\
\text { Genes }\end{array}$ & $\begin{array}{l}\text { Personal Data, Fundus Images, } \\
\text { Genome Data }\end{array}$ & SVM MKL \\
\hline $\begin{array}{l}\text { (Li et al., } \\
\text { 2018)[11] }\end{array}$ & Yes & Yes & Structured & Visual Field Repots & $\begin{array}{l}\text { SVM, RF, K-NN, } \\
\text { CNN }\end{array}$ \\
\hline $\begin{array}{l}\text { (Noronha } \\
\text { et al., } \\
2019)[25]\end{array}$ & Yes & No & Images & Fundus Image & SVM, NB \\
\hline $\begin{array}{l}\text { (Yo and } \\
\text { Hong, } \\
\text { 2015)[28] }\end{array}$ & Yes & No & Structured & Clinical Variables & MLR, ANN \\
\hline $\begin{array}{l}\text { (Li et al., } \\
\text { 2020)[29] }\end{array}$ & Yes & Yes & $\begin{array}{l}\text { Structured } \\
\text { and Images }\end{array}$ & Fundus Image, Medical History Data & RNN(ResNet101) \\
\hline $\begin{array}{l}\text { (Acharya } \\
\text { et al., } \\
\text { 2017)[26] }\end{array}$ & Yes & No & Images & Fundus Images & $\begin{array}{l}\text { DT, QDA, LDA, } \\
\text { SVM, KNN, PNN }\end{array}$ \\
\hline $\begin{array}{l}\text { (Mookiah } \\
\text { et al., } \\
\text { 2012)[27] }\end{array}$ & Yes & Yes & Images & Fundus Images & SVM \\
\hline $\begin{array}{l}\text { (Chai et al., } \\
\text { 2018)[32] }\end{array}$ & Yes & No & $\begin{array}{l}\text { Structured } \\
\text { and Images }\end{array}$ & Fundus Images, Clinical Data & $\begin{array}{l}\text { Multi-Branch } \\
\text { Neural Network }\end{array}$ \\
\hline $\begin{array}{l}\text { (Pathan } \\
\text { et al., } \\
\text { 2021)[33] }\end{array}$ & Yes & Yes & Images & Fundus Images & $\begin{array}{l}\text { ANN, SVM, } \\
\text { AdaBoost }\end{array}$ \\
\hline $\begin{array}{l}\text { (Kim et al., } \\
\text { 2017)[30] }\end{array}$ & Yes & No & Structured & $\begin{array}{l}\text { RNFL Thickness, Visual Field test } \\
\text { Parameter, General Ophthalmic } \\
\text { Examination }\end{array}$ & $\begin{array}{l}\text { RF, DT, SVM, } \\
\text { KNN }\end{array}$ \\
\hline
\end{tabular}

SAP: Standard Automated Perimetry, LDA: Linear Discriminant Analysis, SVM: Support Vector Machine, NB: Naïve Bayes, ANN: Artificial Neural Networks, MKL: Multi Kernel Learning, RF: Random Forest, K-NN: K-Nearest Neighbor, CNN:

Convolutional Neural Networks, MLR: Multi Logistic Regression, RNN: Residual Neural Network, QDA: Quadratic Linear Regression, PNN: Probabilistic Neural Networks, Reg: Regression, RNFL: Retinal Nerve Fiber Layer, IOP:

Intraocular Pressure.

methodology. Figure 1 shows the main steps of this study and the proposed method to predict glaucoma.

All preprocessing, modeling, and evaluation were done in Python language, and visualization was done in Python and Microsoft Excel in this study.

The steps as shown in Fig. 1 are described with more details in the following subsections:

\section{Data description}

Shahroud eye cohort study was started in 2009 to diagnose and detect visual impairments and eye diseases in Shahroud city, Northeast Iran.[34].

In the first phase of the Shahroud eye cohort study, 6311 people aged 40-64 years were selected by random cluster sampling. Among them, 5190 individuals, including 


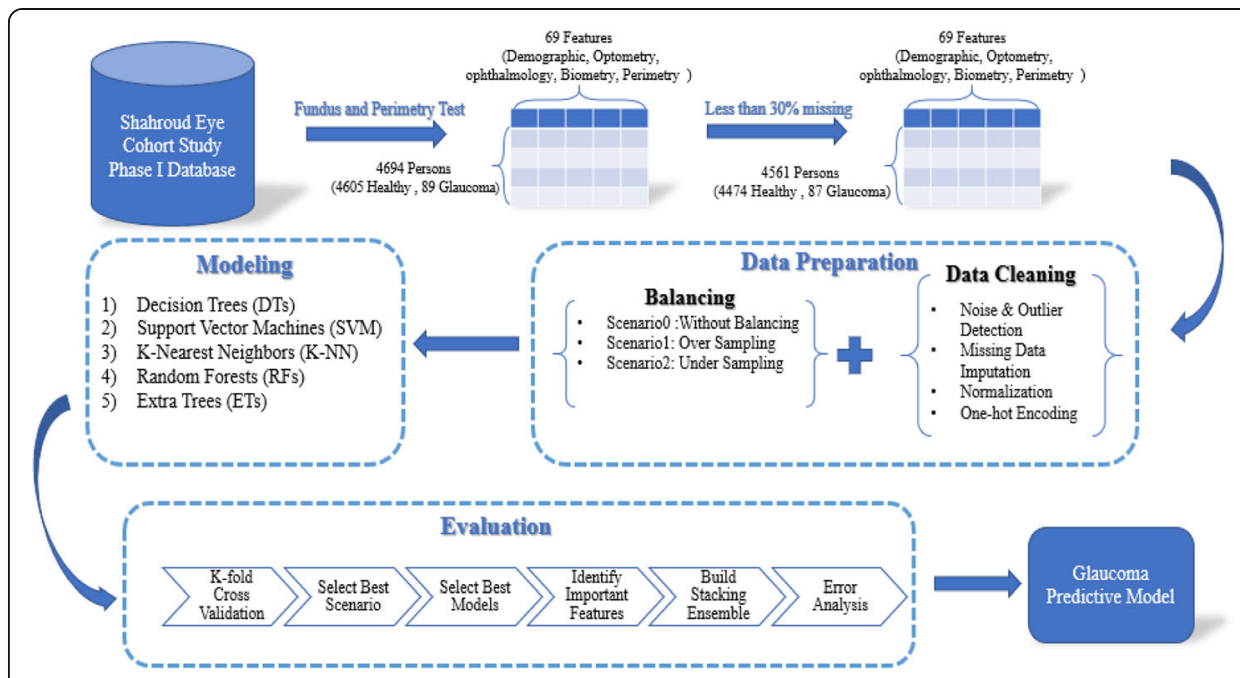

Fig. 1 The main steps of this study proposed method for glaucoma prediction from Shahroud eye cohort study dataset

2990 females, have been participated in the study. Fundus imaging and perimetry examination have been performed for 4694 participants. Assessing fundus and perimetry images by the ophthalmologists for glaucoma detection was performed. According to this study, the prevalence rate of glaucoma was estimated at $1.92 \%$ of the population[5] among the considered persons, eighty-nine participants diagnosed as glaucoma patients.

Table 2 lists the demographic characteristics, optometry, ophthalmology, biometry, and perimetry examinations for the contributors in the first phase of the Shahroud eye cohort study.

However, some participants who have more than $30 \%$ missing values have been eliminated from this study. Therefore, 67 variables describing 4474 non-glaucoma persons and 87 glaucoma persons are considered in this study.

\section{Data preprocessing}

The dataset should be partitioned into two non-overlapping datasets, including training and test datasets. For this purpose, K-fold Cross-Validation (C.V.) was used once with $\mathrm{K}=5$ and $\mathrm{K}=10$. The main steps of data preprocessing and preparation performed in this study were divided into two main categories: data cleaning and balanced sampling. Data cleaning steps are outlier detection and removal, missing value handling, data normalization, and one-hot encoding. Three different scenarios were evaluated and compared for data balancing or not. The first scenario was imbalanced data. The second and third scenarios used over-sampling and under-sampling strategies for balancing the training dataset.

For outlier detection, numerical variables are analyzed using the interquartile range (IQR) as a commonly used outlier detection method[36]. According to this method, no outlier is detected in numerical variables.

Categorical nominal variables were converted to dummy binary variables. Missing value imputation was performed for variables having a missing value rate lower than 
Table $\mathbf{2}$ List of the considered variables in this study

\begin{tabular}{|c|c|c|c|}
\hline Feature Name & Values & Feature Name & Values \\
\hline Sex & $\begin{array}{l}\text { Female/ } \\
\text { Male }\end{array}$ & Age (in Years) & Number \\
\hline Body Mass Index (in kg/m2) & Number & Occupation Status & $\begin{array}{l}\text { Nominal (including six } \\
\text { categories) }\end{array}$ \\
\hline Socioeconomic Status & Number & Marital Status & $\begin{array}{l}\text { Nominal (including four } \\
\text { categories) }\end{array}$ \\
\hline Smoking & Yes/No & Diabetes & Yes/No \\
\hline Diabetes Drug & Yes/No & Iris Color* & $\begin{array}{l}\text { Nominal (including five } \\
\text { categories) }\end{array}$ \\
\hline $\begin{array}{l}\text { Systolic Blood Pressure (in } \\
\mathrm{mm} / \mathrm{Hg} \text { ) }\end{array}$ & Number & Diastolic Blood Pressure (in mm/Hg) & Number \\
\hline Hyper Tension Drug & Yes/No & Intraocular Pressure* (in mm/Hg) & Number \\
\hline Visual Accuity* (in logMAR) & Number & Myopia* & Yes/No \\
\hline Hyperopia & Yes/No & Nuclear Cataract* & $\begin{array}{l}\text { Nominal (including five } \\
\text { categories) }\end{array}$ \\
\hline Glaucoma Drug* & Yes/No & Cortical Cataract* & $\begin{array}{l}\text { Nominal (including five } \\
\text { categories) }\end{array}$ \\
\hline Glaucoma* & Yes/No & Posterior Subcapsular Cataract* & $\begin{array}{l}\text { Nominal (including five } \\
\text { categories) }\end{array}$ \\
\hline Astigmatism* (in Diopter) & Number & Spherical Equivalent*(in Diopter) & Number \\
\hline Angle Closure* & Yes/No & Number of Visual Detect* & Number \\
\hline Vertical Cup to Disk Ratio* & Number & Axial length* (in mm) & Number \\
\hline $\begin{array}{l}\text { Anterior Chamber Depth* } \\
(\mathrm{mm})\end{array}$ & Number & Corneal Thickness *(in mm) & Number \\
\hline Lens Thickness* (in mm) & Number & $\begin{array}{l}\text { Corneal White to White Diameter* } \\
\text { (in } \mathrm{mm} \text { ) }\end{array}$ & Number \\
\hline Corneal Radius, Flat* (in mm) & Number & Corneal Radius, Steep* (in mm) & Number \\
\hline Keratometry* (K1) & Number & Keratometry* (K2) & Number \\
\hline Iris Barycentric-X-Coordinate* & Number & Iris Barycentric-Y-Coordinate* & Number \\
\hline $\begin{array}{l}\text { Pupil Barycentric-X- } \\
\text { Coordinate* }^{*}\end{array}$ & Number & Pupil Barycentric-Y-Coordinate* & Number \\
\hline Pupil Distance*(in mm) & Number & & \\
\hline
\end{tabular}

"Stars show that the feature has been measured in both eyes.

$30 \%$, and other variables were excluded from the study. Missing values were replaced with mean and mode for numerical variables and binary variables, respectively.

Features in our analysed dataset have different units or come from different examinations, and features have different value range. Some learning algorithms, similar distance-based methods like K-Nearest Neighbors (K-NN) or kernel machines like Support-Vector Machines (SVM), are sensitive to features ranges. This sensitivity can cause models bias to features with higher variations. Data normalization was applied to the Min-Max normalization method to avoid dominating variables with a low, extensive range of variations and improve evaluation metrics.

Since our analyzed dataset's glaucoma prevalence rate was about $1.9 \%$, the class distribution was significantly imbalanced. On the other hand, more than $92 \%$ of the considered persons belong to the non-glaucoma group (majority class), and only $1.9 \%$ of the persons are assigned to the glaucoma class (minor class). The previous studies have shown that the classifiers trained on imbalanced datasets can have higher accuracy for 
classifying the major class. However, the minor class cannot be trained with high accuracy [37].

To overcome the imbalance distribution in this study, dataset between the classes, three different strategies applied were under-sampling, over-sampling, and combining over-sampling and under-sampling strategy used in the previous studies [37].

In this study, three different scenarios were designed and compared to address the imbalance dataset's challenges. The first was sampling from data without balancing the class distribution (Scenario 0). The second was uses over-sampling from the minor class (Scenario1). The last was the balanced bagging ensemble method, which has been proposed to overcome the imbalance dataset challenges in a previous study (Scenario 2) [38]. The bagging ensemble using a high number of estimators can guarantee that the major class observations contribute to training one of the estimators.

\section{Modeling}

Classifiers considered in this study include well-known classifier Decision Trees (DT) [39], Support Vector Machines, and ensemble classifiers having DT as their base classifiers, such as Extra Tree (ET) [40] and Random Forests (RF) [41]. These classifiers have different advantages and were used as a base classifier in three scenarios. For tuning the hyperparameters of the classifiers and choosing the resampling ratio, the Grid search method was used in this study. For DT and the ensemble based on DT like RF and ET, the splitting criterion was the 'Information Gain,' and the number of trees was 200. The kernel function of SVMs was 'Radial Basis Function (RBF)' in Scenario1 and Scenario2, and Polynomial' in Senario3. The number of neighbours in the K-NN was seven, and the distance metric was 'Euclidean.' In Scenario2, the oversampling ratio was different for each classifier and was determined by the Grid search. In Scenario3, the number of estimators of the balanced bagging ensemble method was 300. This number of the base estimator guarantee that every sample in the training set contributes at least to train one of the estimators and avoid overfitting.

\section{Evaluation}

As illustrated in Fig. 1, evaluation tasks include the K-fold C.V. strategy for sampling from data, choosing the best scenario for handling imbalanced data, choosing the best classifiers, identifying the important features, building the proposed stacking ensemble model, and error analysis.

As mentioned in the preprocessing subsection, data was partitioned into training and test datasets based on the K-fold C.V. strategy for $\mathrm{K}=5$ and $\mathrm{K}=10$. The best scenario for handling imbalanced data and classifiers was chosen by comparing different combinations of the scenarios and classifiers based on their performance on the validation dataset. Then, important features ranked with the best classifiers were identified. Afterward, the proposed two-layered stacking ensemble classifier was built in which the first layer included different classifiers and the second layer used a logistic regression model.

For error analysis, evaluating and comparing the classifiers and different scenarios used for balanced sampling, various standard performance measures which were 
calculated and reported were accuracy sensitivity, specificity, F1-Score, and area under receiver operating characteristics (ROC) curve (AUC) as Eqs. (1)-(4).

$$
\begin{aligned}
& \text { Accuracy }=\frac{T P+T N}{T P+T N+F P+F N} \\
& \text { Sensitivity }=\frac{T P}{T P+F N} \\
& \text { Specificity }=\frac{T N}{F P+T N} \\
& F 1-\text { score }=\frac{T P}{T P+\frac{(F P+F N)}{2}}
\end{aligned}
$$

\section{Experimental results}

For evaluating the proposed scenarios, every classifier in each scenario was executed 30 times, and the performance measures, including accuracy, sensitivity, specificity, and F1-Score, are reported in Table 3.

As illustrated by Table 3, the last scenario's classifiers had better performance for predicting the non-glaucoma class and glaucoma class. Decision trees and random forests which outperform the other classifiers were contributed to the balanced bagging ensemble as its base classifiers.

The ROC curves are illustrated in Fig. 2 to compare different scenarios and classifiers.

According to DT and RF's reasonable accuracy in the last scenario, top-ranked features were selected based on the feature importance scores assigned to the variables with DT and RF. 10-top ranked variables in the first quartile for glaucoma prediction from the Shahroud eye cohort dataset based on their average score on 300 executions of the classifiers are listed in Table 4.

As illustrated by Table 4, eight variables among 10-top ranked features identified by DT and RF are common, and they include NVD, VCDR, WTW, Systolic BP, PCY, AST, Age, and AL, which come from the different examination sources.

Finally, a novel two-layered stacking ensemble classifier is proposed in which the first layer combines two superior classifiers of the last scenario and the second layer uses logistic regression. Table 5 shows the performance measures of the proposed stacking ensemble classifier for Glaucoma prediction.

\section{Discussion}

For assessing the performance of the proposed stacking ensemble classifier in this study, 30 executions of 10 -fold C.V. are analyzed. According to the experimental results, 3200 persons belonging to the non-glaucoma class (71.5\%) and 59 glaucoma persons $(67.8 \%)$ are correctly predicted in all executions. On the other hand, 368 persons of non-glaucoma class $(8.2 \%)$ and glaucoma class $(8 \%)$ are misclassified in all executions. Figure 3 indicates how many times of the executions each instance is predicted correctly.

According to Fig. 3, a novel confusion matrix (NCM) is proposed based on the thresholds for at least $0 \%, 30 \%, 60 \%, 90 \%$, and $100 \%$ instances that could be 
Table 3 Comparing the performance of the classifiers in each scenario

\begin{tabular}{|c|c|c|c|c|c|}
\hline \multirow[t]{2}{*}{ Model } & $\begin{array}{l}\% \text { Acc } \\
(\text { Mean } \pm \text { std) }\end{array}$ & $\begin{array}{l}\% \text { Spe } \\
(\text { Mean } \pm \text { std) }\end{array}$ & $\begin{array}{l}\% \text { Sen } \\
(\text { Mean } \pm \text { std) }\end{array}$ & $\begin{array}{l}\text { \%Sen } \\
\text { (Cl } 95 \%)\end{array}$ & $\begin{array}{l}\text { \%F_score } \\
\text { (Mean } \pm \text { std) }\end{array}$ \\
\hline & \multicolumn{5}{|l|}{ Scenario0_5fold } \\
\hline DT & $96.89 \pm 0.45$ & $98.35 \pm 0.45$ & $21.84 \pm 9.32$ & $(20.33,23.35)$ & $20.87 \pm 8.20$ \\
\hline SVM & $98.08 \pm 0.07$ & $99.99 \pm 0.04$ & $00 \pm 00$ & $(00,00)$ & $00 \pm 00$ \\
\hline KNN & $97.07 \pm 0.34$ & $98.91 \pm 0.35$ & $2.30 \pm 3.46$ & $(1.74,2.86)$ & $2.80 \pm 4.23$ \\
\hline RF & $98.09 \pm 0.05$ & $100 \pm 00$ & $0.04 \pm 0.48$ & $(00,0.12)$ & $0.07 \pm 0.90$ \\
\hline ET & $98.08 \pm 0.07$ & $99.99 \pm 0.04$ & $0.19 \pm 1.04$ & $(00,00)$ & $0.36 \pm 1.95$ \\
\hline \multicolumn{6}{|c|}{ Scenario0_10fold } \\
\hline DT & $96.90 \pm 00.71$ & $98.31 \pm 00.68$ & $24.86 \pm 13.89$ & $(23.28,26.44)$ & $23.24 \pm 12.41$ \\
\hline SVM & $98.06 \pm 00.00$ & $99.98 \pm 00.06$ & $00 \pm 00$ & $(00,00)$ & $00 \pm 00$ \\
\hline KNN & $97.04 \pm 00.53$ & $98.82 \pm 0.53$ & $02.25 \pm 04.83$ & $(1.70,2.80)$ & $2.66 \pm 5.76$ \\
\hline $\mathrm{RF}$ & $98.09 \pm 0.10$ & $100 \pm 00$ & $0.04 \pm 0.64$ & $(0.00,0.11)$ & $0.07 \pm 1.15$ \\
\hline ET & $98.08 \pm 0.13$ & $99.98 \pm 0.06$ & $0.27 \pm 1.74$ & $(0.07,0.47)$ & $0.48 \pm 3.12$ \\
\hline \multicolumn{6}{|c|}{ Scenario1_5fold } \\
\hline DT & $96.89 \pm 0.48$ & $98.35 \pm 00$ & $21.57 \pm 9.53$ & $(20.02,23.11)$ & $20.74 \pm 8.28$ \\
\hline SVM & $97.61 \pm 0.31$ & $99.16 \pm 0.33$ & $17.94 \pm 8.33$ & $(16.59,19.29)$ & $21.75 \pm 8.72$ \\
\hline KNN & $94.60 \pm 0.61$ & $96.23 \pm 0.61$ & $10.97 \pm 7.23$ & $(9.80,12.14)$ & $7.15 \pm 4.72$ \\
\hline RF & $98.08 \pm 0.06$ & $99.99 \pm 0.04$ & $00 \pm 00$ & $(00,00)$ & $00 \pm 00$ \\
\hline ET & $98.09 \pm 0.06$ & $100 \pm 0.00$ & $00 \pm 00$ & $(00,00)$ & $00 \pm 00$ \\
\hline \multicolumn{6}{|c|}{ Scenario1_10fold } \\
\hline DT & $96.81 \pm 0.67$ & $98.27 \pm 0.64$ & $21.82 \pm 13.3$ & $(20.31,23.33)$ & $20.39 \pm 11.99$ \\
\hline SVM & $97.65 \pm 0.47$ & $99.21 \pm 0.43$ & $17.61 \pm 12.1$ & $(16.23,18.99)$ & $21.48 \pm 13.61$ \\
\hline KNN & $94.60 \pm 0.90$ & $96.23 \pm 0.91$ & $10.99 \pm 10.07$ & $(9.84,12.14)$ & $7.13 \pm 6.48$ \\
\hline RF & $98.08 \pm 0.12$ & $99.99 \pm 0.05$ & $0.08 \pm 0.96$ & $(0.00,0.19)$ & $0.14 \pm 1.72$ \\
\hline ET & $98.09 \pm 0.10$ & $100 \pm 00$ & $00 \pm 00$ & $(00,00)$ & $00 \pm 00$ \\
\hline \multicolumn{6}{|c|}{ Scenari2_5fold } \\
\hline DT & $87.40 \pm 1.49$ & $87.65 \pm 1.58$ & $74.41 \pm 9.25$ & $(72.91,75.91)$ & $18.47 \pm 2.27$ \\
\hline SVM & $83.52 \pm 1.75$ & $83.38 \pm 1.79$ & $65.35 \pm 9.86$ & $(63.75,66.94)$ & $13.22 \pm 2.16$ \\
\hline KNN & $80.94 \pm 1.59$ & $81.80 \pm 1.63$ & $36.26 \pm 10.05$ & $(34.64,37.89)$ & $6.77 \pm 1.81$ \\
\hline RF & $88.90 \pm 1.08$ & $89.24 \pm 1.13$ & $71.39 \pm 8.96$ & $(69.93,72.84)$ & $19.75 \pm 2.42$ \\
\hline ET & $92.75 \pm 0.91$ & $93.49 \pm 0.91$ & $54.66 \pm 10.67$ & $(52.94,56.39)$ & $22.43 \pm 4.41$ \\
\hline \multicolumn{6}{|c|}{ Scenario2_10fold } \\
\hline DT & $87.61 \pm 1.67$ & $87.88 \pm 1.72$ & $73.80 \pm 14.38$ & $(72.16,75.44)$ & $18.61 \pm 3.70$ \\
\hline SVM & $83.69 \pm 1.80$ & $84.02 \pm 1.83$ & $66.71 \pm 15.36$ & $(64.96,68.46)$ & $13.55 \pm 3.16$ \\
\hline KNN & $81.00 \pm 1.87$ & $81.84 \pm 1.91$ & $37.69 \pm 15.55$ & $(35.90,39.44)$ & $7.02 \pm 2.88$ \\
\hline RF & $88.87 \pm 1.48$ & $89.10 \pm 1.51$ & $72.35 \pm 13.67$ & $(70.80,73.91)$ & $19.87 \pm 4.00$ \\
\hline ET & $92.68 \pm 1.16$ & $93.40 \pm 1.15$ & $55.55 \pm 16.60$ & $(53.66,57.44)$ & $22.54 \pm 6.68$ \\
\hline
\end{tabular}

Acc: Accuracy; Spe: Specificity; Sen: Sensitivity; Cl: Confidence Interval

predicted correctly, as shown in Table 6. For example, if the threshold is $60 \%$, TP and TN show that the instances are correctly classified into positive (Glaucoma) and negative (non-glaucoma) at least 18 times of 30 executions, respectively.

The results shown in Table 5 are similar to the results corresponding to the threshold of $60 \%$ in Table 6. According to the experimental results described in this section, data instances that no classifier can correctly predict negatively impact the classifier performance. 

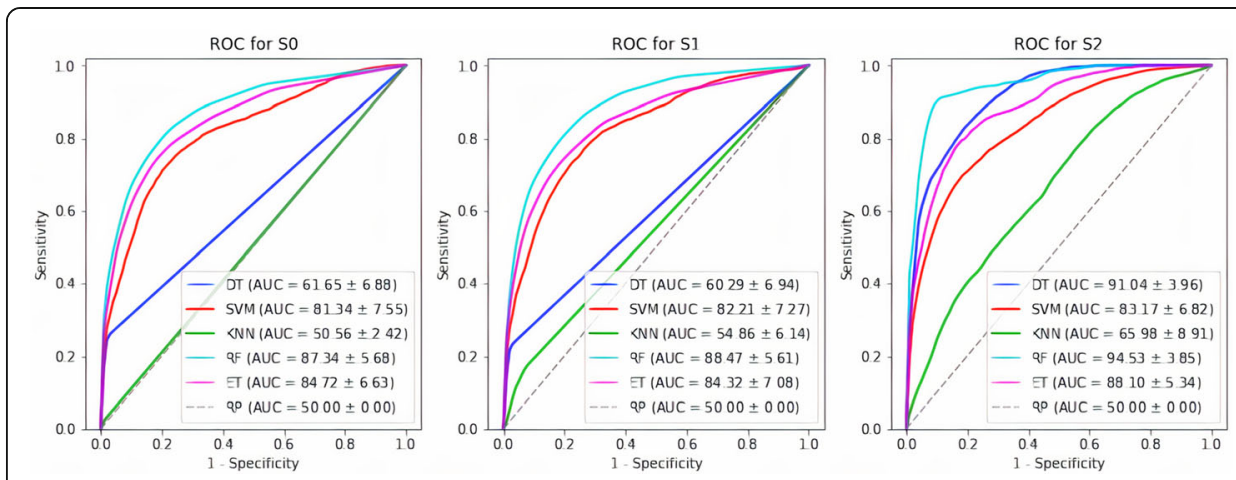

Fig. 2 ROC curve for models in each scenario

FP instances are the data instances with glaucoma, but the classifier has misclassified them as the non-glaucoma class label.

The average of top-ranked features for non-glaucoma and glaucoma classes is compared using the t-student test shown in Table 7 to investigate the significant difference between the non-glaucoma and glaucoma classes per each top-ranked feature.

As listed in Table 7, the average of LT, Spherical Equivalent, AST, Systolic BP, AL, Age, VCD, and NVD are significantly different for glaucoma and non-glaucoma groups. It indicates that the mentioned variables can distinguish well two classes in our study. However, the average of WTW, PCY, BMI, and Diastolic BP is not significantly different for the glaucoma and non-glaucoma groups.

\section{Conclusions}

Early identification of the persons with a high risk of glaucoma can help early beginning the necessary treatment and monitoring disease and prevent converting disease to the acute form. In this study, a novel stacking ensemble classifier composed of several machine learning classifiers is proposed, designed, and used for glaucoma prediction considering the Shahroud eye cohort dataset. This study's input variables and predictors for glaucoma prediction are demographic characteristics, ophthalmology features,

Table 4 Feature importance in the best models

\begin{tabular}{lll}
\hline Rank & Decision Tree & Random Forest \\
\hline 1 & NVD & NVD \\
2 & VCDR & VCDR \\
3 & Age & AL \\
4 & AL & Systolic BP \\
5 & WTW & Age \\
6 & Systolic BP & AST \\
7 & PCY & BMl \\
8 & Spherical Equivalent & Diastolic BP \\
9 & LT & PCY \\
10 & AST & WTW \\
\hline
\end{tabular}

NVD: Number of Visual Detect; VCDR: Vertical Cup to Disk Ratio; AL: Axial Length; WTW: Corneal Whit to White Diameter; PCY: Posterior Capsule; LT: Lens Thickness; AST: Astigmatism, BMI: Body Mass Index; BP: Blood Pressure 
Table 5 Performance of stacking models

\begin{tabular}{llllll}
\hline & $\begin{array}{l}\text { \%Acc } \\
\text { (Mean } \pm \mathbf{s t d})\end{array}$ & $\begin{array}{l}\text { \%Spe } \\
(\text { Mean } \pm \text { std) }\end{array}$ & $\begin{array}{l}\text { \%Sen } \\
\text { (Mean } \pm \text { std) }\end{array}$ & $\begin{array}{l}\text { \%Sen } \\
(\mathbf{C l} \text { 95 \%) }\end{array}$ & $\begin{array}{l}\text { \%F_score } \\
\text { (Mean } \pm \text { std) }\end{array}$ \\
\hline $\begin{array}{l}\text { Stacking Ensemble trained with all } \\
\text { features }\end{array}$ & $83.56 \pm 1.35$ & $82.21 \pm 1.75$ & $81.32 \pm 10.39$ & $(80.07$, & $80.98 \pm 6.65$ \\
$\begin{array}{l}\text { Stacking ensemble trained with } \\
\text { top-ranked features }\end{array}$ & $83.01 \pm 1.98$ & $83.00 \pm 2.10$ & $83.17 \pm 12.03$ & $(81.80,84.54)$ & $82.58 \pm 6.34$ \\
\hline
\end{tabular}

biometry, and perimetry descriptors for persons aged between 40 and 64 years old in Shahroud. Three scenarios are compared for handling an imbalanced dataset. The experimental results show that balanced bagging based on random forests and decision trees can improve the sensitivity and performance of glaucoma prediction with the average accuracy of 87.61 and 88.87 , the sensitivity of 73.80 and 72.35 , specificity of 87.88 and 89.10, and AUC of 91.04 and 94.53, respectively. On the other hand, the proposed stacking ensemble classifier achieves an average accuracy of 83.56, a sensitivity of 82.21, a specificity of 81.32, and an AUC of 88.54.

The previous studies used three different data types: fundus images, genome data, and structured data to develop a glaucoma prediction and diagnosis model. These studies achieved high-performance measures on fundus images or the combination of different data types, as shown in Table 8. This study used extensive ophthalmologic examinations like biometry, perimetry, and some clinical data to develop the predictive glaucoma model without fundus images or genome data. The developed model in this study has lower performance measures against other studies. Still, it has less complexity and cost and can use as the base of a decision support system in clinics to diagnose and screen glaucoma.

Top-ranked features for predicting glaucoma identified using DT and RF are listed in Table 4. These top features come from different eye examinations like perimetry and biometry or demographic features that can measure in every clinic. As discussed in the introduction, some of these top features are the main risk factors of glaucoma diseases like age, BMI, blood pressure, and axial length, identified in many studies. On the other hand, some of these features like NVD and VCDR are used to identify glaucoma by physicians instantly. As mentioned in a previous study[5], top-ranked features for glaucoma prediction determined using simple and multivariate logistic regression have been

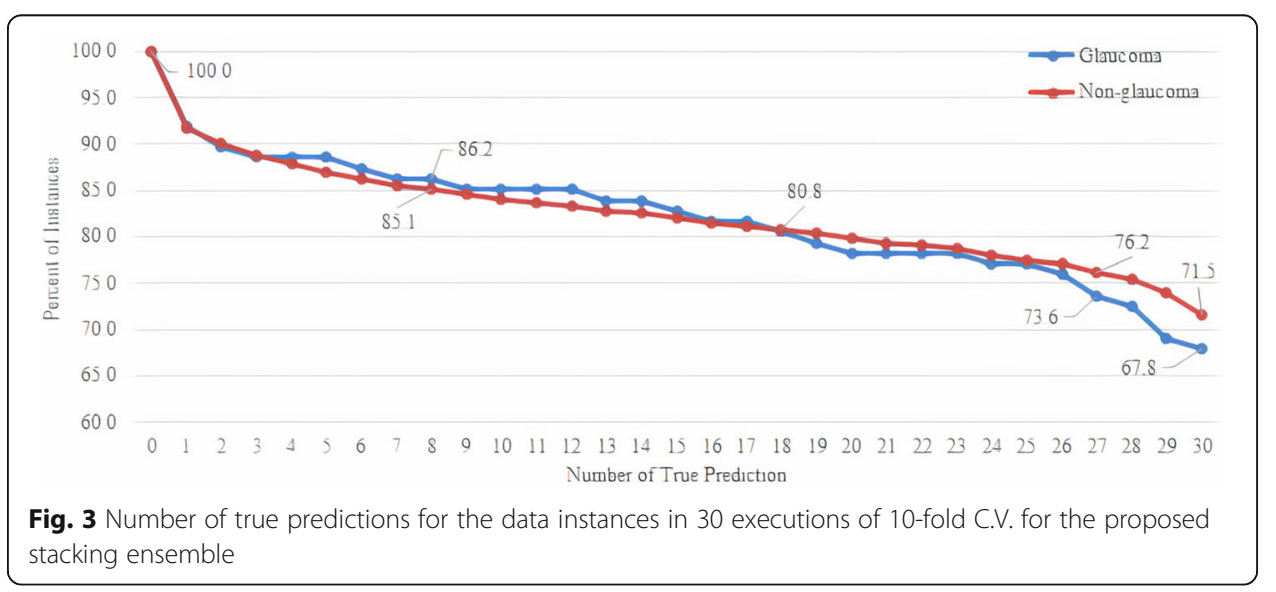


Table 6 The novel proposed confusion matrix (NCM) for our proposed stacking ensemble method in this study

\begin{tabular}{llllllll}
\hline Threshold & TP & TN & FN & FP & \%Acc & \%Sen & \%Spe \\
\hline 0 & 80 & 4106 & 7 & 368 & 91.78 & 91.95 & 91.77 \\
30 & 74 & 3784 & 13 & 690 & 84.59 & 85.06 & 84.58 \\
60 & 70 & 3614 & 17 & 860 & 80.77 & 80.46 & 80.78 \\
90 & 64 & 3409 & 23 & 1065 & 76.15 & 73.56 & 76.20 \\
100 & 59 & 3200 & 28 & 1274 & 71.45 & 67.82 & 71.52 \\
\hline
\end{tabular}

age, IOP, sex, diabetes, myopia, and axial length. The Van diagram in Fig. 4 shows the top predictors of glaucoma.

This study aims to discriminate between glaucoma patients and non-glaucoma persons. The proposed and designed models in this study are disable to diagnose glaucoma type. Different types of glaucoma can be discriminated against, predicted, and diagnosed using machine learning models as a future research direction. Top-ranked features and risk factors for each type of glaucoma can be identified.

The first phase of the Shahroud Eye Cohort Study was used to predict glaucoma with extensive ophthalmologic examinations and demographic data without any fundus images and achieve an average accuracy of 83.56. The Shahroud Eye Cohort Study was conducted in two more phases with an interval of five years. For future work, the glaucoma condition of participating in the second phase can be use as the label for the first

Table 7 Comparing the average of top-ranked features for Non-glaucoma and Glaucoma classes using t-student test

\begin{tabular}{llll}
\hline Feature & Glaucoma & Non-glaucoma & P-value \\
\hline NUMBEROFVISUALDEFECTOS & 17.06 & 3.34 & $<0.001$ \\
NUMBEROFVISUALDEFECTOD & 18.75 & 2.48 & $<0.001$ \\
Vertical CD Ratio Right & 0.28 & 0.17 & $<0.001$ \\
Vertical CD Ratio Left & 0.28 & 0.17 & $<0.001$ \\
Age & 53.33 & 50.08 & $<0.001$ \\
AL Left & 23.51 & 23.04 & $<0.001$ \\
AL Right & 23.56 & 23.06 & $<0.001$ \\
Systolic BP & 130.77 & 128.02 & $<0.001$ \\
Diastolic BP & 79.79 & 79.44 & 0.403 \\
AST Left & 0.95 & 0.82 & $<0.001$ \\
AST Right & 0.98 & 0.82 & $<0.001$ \\
BMI & 28.3 & 28.45 & 0.415 \\
PCY Left & -0.04 & -0.02 & 0.007 \\
PCY Right & -0.01 & 0.01 & 0.002 \\
WTW Right & 11.8 & 11.78 & 0.258 \\
WTW Left & 11.79 & 11.8 & 0.502 \\
Spherical Equivalent & -0.69 & 0.1 & $<0.001$ \\
LT Left & 4.29 & 4.24 & $<0.001$ \\
LT Right & 4.31 & 4.26 & $<0.001$ \\
\hline AL:Axia Lent & & &
\end{tabular}

AL: Axial Length, BP: BP: Blood Pressure, AST: Astigmatism, BMI: Body Mass Index, WTW: White to White distance, LT: Lenz Thickness 
Table 8 Comparing the performance of the proposed method in this study with the previous studies

\begin{tabular}{|c|c|c|c|}
\hline Authors & Dataset & Classifier & $\begin{array}{l}\text { Best Performance } \\
\text { Measures (\%) }\end{array}$ \\
\hline $\begin{array}{l}\text { (Li et al., } \\
\text { 2019)[31] }\end{array}$ & SAP Data & LDA, SVM, NB, ANN & $\mathrm{AUC}=91.2$ \\
\hline $\begin{array}{l}\text { (Liu et al., } \\
\text { 2013)[10] }\end{array}$ & $\begin{array}{l}\text { Personal Data, Fundus Images, Genome } \\
\text { Data }\end{array}$ & SVM MKL & $A \cup C=86.6$ \\
\hline $\begin{array}{l}\text { (Li et al., } \\
\text { 2018)[11] }\end{array}$ & Visual Field Repots & SVM, RF, K-NN, CNN & $\begin{array}{l}\text { Acc }=87.6, \text { Sen }=93.2 \\
\text { Spe }=82.6\end{array}$ \\
\hline $\begin{array}{l}\text { (Noronha et al., } \\
\text { 2019)[25] }\end{array}$ & Fundus Image & SVM, NB & $\begin{array}{l}\text { Acc }=92.65, \text { Sen }= \\
100, \text { Spe }=92.0\end{array}$ \\
\hline $\begin{array}{l}\text { (Yo and Hong, } \\
\text { 2015)[28] }\end{array}$ & Clinical Variables & MLR, ANN & $\begin{array}{l}\text { Acc }=84.0, \text { Sen }=78.3 \\
\text { Spe }=85.9\end{array}$ \\
\hline $\begin{array}{l}\text { (Li et al., } \\
\text { 2020)[29] }\end{array}$ & Fundus Image, Medical History Data & RNN(ResNet101) & $\begin{array}{l}\text { Acc }=96.5, \text { Sen }=99.8, \\
\text { Spe }=99.9\end{array}$ \\
\hline $\begin{array}{l}\text { (Kim et al., } \\
\text { 2017)[30] }\end{array}$ & $\begin{array}{l}\text { RNFL Thickness, VF test Parameter, } \\
\text { General Ophthalmic Examination }\end{array}$ & RF, DT, SVM, KNN & $\begin{array}{l}\text { Acc }=98, \text { Sen }=98.3 \\
\text { Spe }=97.5\end{array}$ \\
\hline $\begin{array}{l}\text { (Acharya et al., } \\
\text { 2017)[26] }\end{array}$ & Fundus Images & $\begin{array}{l}\text { DT, QDA, LDA, SVM, } \\
\text { KNN, PNN }\end{array}$ & $A C C=95.8$ \\
\hline $\begin{array}{l}\text { (Mookiah et al., } \\
\text { 2012)[27] }\end{array}$ & Fundus Images & SVM & $\begin{array}{l}\text { Acc }=95.0, \text { Sen }=93.33, \\
\text { Spe }=96.67\end{array}$ \\
\hline $\begin{array}{l}\text { (Chai et al., } \\
\text { 2018)[32] }\end{array}$ & Fundus Images, Clinical Data & $\begin{array}{l}\text { Multi Branch Neural } \\
\text { Network }\end{array}$ & $\begin{array}{l}\text { Acc }=99.24, \text { Sen }= \\
97.91, \text { Spe }=93.59\end{array}$ \\
\hline $\begin{array}{l}\text { (Pathan et al., } \\
\text { 2021)[33] }\end{array}$ & Fundus Images & ANN, SVM, AdaBoost & $\begin{array}{l}\text { Acc }=98.0, \text { Sen }=100 \\
\text { Spe }=97.0\end{array}$ \\
\hline This study & $\begin{array}{l}\text { Extensive ophthalmologic examination } \\
\text { and clinical data }\end{array}$ & $\begin{array}{l}\text { DT, RF, ET, KNN, SVM, } \\
\text { Stacking Ensemble }\end{array}$ & $\begin{array}{l}\text { Acc }=83.56, \text { Sen }= \\
82.21, \text { Spe }=81.32\end{array}$ \\
\hline
\end{tabular}

SAP: Standard Automated Perimetry, LDA: Linear Discriminant Analysis, SVM: Support Vector Machine, NB: Naïve Bayes, ANN: Artificial Neural Networks, MKL: Multi Kernel Learning, RF: Random Forest, K-NN: K-Nearest Neighbor, CNN: Convolutional Neural Networks, MLR: Multi Logistic Regression, RNN: Residual Neural Network, QDA: Quadratic Linear Regression, PNN: Probabilistic Neural Networks, Reg: Regression, RNFL: Retinal Nerve Fiber Layer, IOP: Intraocular Pressure.

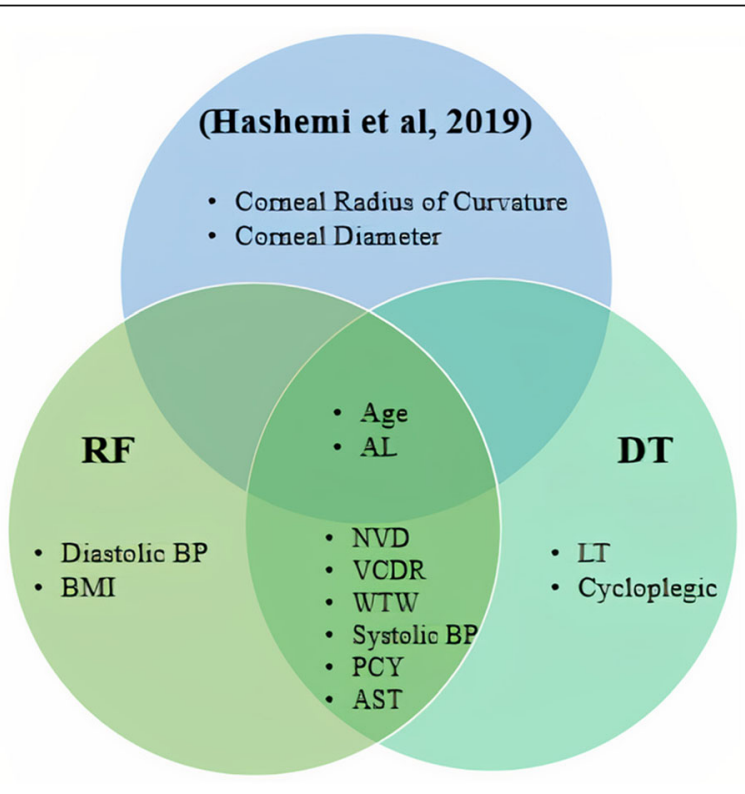

Fig. 4 The Van diagram indicating our top-ranked features identified using DT and RF and top-ranked features identified in a previous study 
phase to develop a prognosis model which can identify people with glaucoma five years earlier and evaluate the model on the third phase.

This study has some main differences compared to the previous related works. In this study, different ophthalmological features are used as the input variables of our models, such as optometric examination results, biometric and perimetric features, ophthalmologic examinations. Moreover, top-ranked features include the variables describing ophthalmologic examination results. Using longitudinal data collected for 5 -years provide us to assess the future trends and changes for glaucoma in people contributing to the cohort study.

\begin{abstract}
Abbreviations
Acc: Accuracy; AUC: Area Under the Curve; ANN: Artificial Neural Networks; AST: Astigmatism; AL: Axial Length; BP: Blood Pressure; BMI: Body Mass Index; CAD: Computer-Assisted Diagnosis; Cl: Confidence Interval; CNN: Convolutional Neural Networks; WTW: Whit to White Diameter; CRISP-DM: Cross-Industry Standard Process for Data Mining; C.V: Cross-Validation; DSS: Decision Support System; DT: Decision Tree; ET: Extra Trees; FN: False Negative; FP: False Positive; IOP: Interocular Pressure; IQR: Interquartile Range; K-NN: K-Nearest Neighbors; LT: Lens Thickness; LDA: Linear Discriminant Analysis; MSVI: Moderate and Severe Vision Impairment; MKL: Multi Kernel Learning; MLR: Multi Logistic Regression; NB: Naïve Bayes; NCM: Novel Confusion Matrix; NVD: Number of Visual Detect; PCY: Posterior Capsule; PNN: Probabilistic Neural Networks; QDA: Quadratic Linear Regression; RBF: Radial Basis Function; RF: Random Forest; ROC: Receiver Operating Characteristics; Reg: Regression; RNN: Residual Neural Network; RNFL: Retinal Nerve Fiber Layer; Sen: Sensitivity; Spe: Specificity; SAP: Standard Automated Perimetry; SVM: Support Vector Machines; TN: True Negative; TP: True Positive; VCDR: Vertical Cup to Disk Ratio
\end{abstract}

\title{
Acknowledgements
}

Not applicable.

\section{Authors' contributions}

Conceptualization: MS, TK and SS. Data curation: MS, TK and SS and MHE. Formal analysis: MS, TK and MHE. Funding acquisition: there is no funding. Investigation: TK and MHE. Methodology: MS and TK. Project administration: TK. Software: MS. Supervision: TK and SS. Validation: TK, MHE, HH and AF. Visualization: MS. Writing - original draft: MS and TK. Writing - review \& editing: TK, MHE, HH and AF. All authors read and approved the manuscript.

\section{Funding}

This study was not funded by any organization.

\section{Availability of data and materials}

This is a retrospective study. Our considered dataset is a cohort dataset (Shahroud Eye Cohort Study). For. access to this dataset, legal procedure should be taken and written and signed commitment form should be. filled.

\section{Declarations}

\section{Ethics approval and consent to participate}

We analyzed a cohort dataset (Shahroud Eye Cohort Study) which has been collected for the previous studies. Therefore, ethics approval and consent for participation is not applicable for this study.

\section{Consent for publication}

All authors have consent for publication.

\section{Competing interests}

The authors declare that there are no conflicts of interest.

\section{Author details}

${ }^{1}$ School of Industrial and Systems Engineering, Tarbiat Modares University, Tehran, Iran. ${ }^{2}$ Ophthalmic Epidemiology Research Center, Shahroud University of Medical Sciences, Shahroud, Iran. ${ }^{3}$ Centre for Analytics and Artificial Intelligence Engineering, University of Toronto, Toronto, Canada. ${ }^{4}$ Noor Ophthalmology Research Center, Noor Eye Hospital, Tehran, Iran. ${ }^{5}$ Department of Epidemiology and Biostatistics, School of Public Health, Tehran University of Medical Sciences, Tehran, Iran.

Received: 29 July 2021 Accepted: 31 October 2021

Published online: 24 November 2021

\section{References}

1. Adelson, J.D., et al., Causes of blindness and vision impairment in 2020 and trends over 30 years, and prevalence of avoidable blindness in relation to VISION 2020: the Right to Sight: an analysis for the Global Burden of Disease Study. The Lancet Global Health, 2020.

2. Flaxman SR, et al. Global causes of blindness and distance vision impairment 1990-2020: a systematic review and metaanalysis. The Lancet Global Health. 2017;5(12):e1221-34. 
3. Tham Y-C, et al. Global prevalence of glaucoma and projections of glaucoma burden through 2040: a systematic review and meta-analysis. Ophthalmology. 2014;121(11):2081-90.

4. McMonnies CW. Glaucoma history and risk factors. Journal of optometry. 2017;10(2):71-8.

5. Hashemi $\mathrm{H}$, et al. Prevalence and risk factors of glaucoma in an adult population from Shahroud, Iran. Journal of Current Ophthalmology. 2019;31(4):366-72.

6. Budenz DL, et al. Prevalence of glaucoma in an urban West African population: the Tema Eye Survey. JAMA ophthalmology. 2013;131(5):651-8.

7. Zhou M, et al. Diabetes mellitus as a risk factor for open-angle glaucoma: a systematic review and meta-analysis. PloS one. 2014;9(8):e102972.

8. Amini, H., et al., The prevalence of glaucoma in Tehran, Iran. 2007.

9. Pakravan M, et al. A population-based survey of the prevalence and types of glaucoma in central Iran: the Yazd eye study. Ophthalmology. 2013;120(10):1977-84.

10. Liu J, et al. Automatic glaucoma diagnosis through medical imaging informatics. Journal of the American Medical Informatics Association. 2013;20(6):1021-7.

11. Li F, et al. Automatic differentiation of Glaucoma visual field from non-glaucoma visual filed using deep convolutional neural network. BMC medical imaging. 2018;18(1):35.

12. Jiang, F., et al., Artificial intelligence in healthcare: past, present and future. Stroke and vascular neurology, 2017. 2(4).

13. Reddy S, Fox J, Purohit MP. Artificial intelligence-enabled healthcare delivery. Journal of the Royal Society of Medicine. 2019;112(1):22-8.

14. Kapoor R, Walters SP, Al-Aswad LA. The current state of artificial intelligence in ophthalmology. Survey of ophthalmology. 2019;64(2):233-40.

15. Gargeya R, Leng T. Automated identification of diabetic retinopathy using deep learning. Ophthalmology. 2017;124(7):962-9.

16. Zhou Y, Li G, Li H. Automatic Cataract Classification Using Deep Neural Network With Discrete State Transition. IEEE Transactions on Medical Imaging. 2019;39(2):436-46.

17. Soliman M, Nasraoui O, Cooper NG. Building a glaucoma interaction network using a text mining approach. BioData mining. 2016;9(1):17.

18. Schmidt-Erfurth $U$, et al. Prediction of individual disease conversion in early AMD using artificial intelligence. Investigative ophthalmology \& visual science. 2018;59(8):3199-208.

19. Yousefi S, et al. Glaucoma progression detection using structural retinal nerve fiber layer measurements and functional visual field points. IEEE Transactions on Biomedical Engineering. 2013;61(4):1143-54.

20. Yousefi, S., et al., Unsupervised Gaussian mixture-model with expectation maximization for detecting glaucomatous progression in standard automated perimetry visual fields. Translational Vision Science \& Technology, 2016. 5(3): p. 2-2.

21. Yousefi $S$, et al. Detection of longitudinal visual field progression in glaucoma using machine learning. American journal of ophthalmology. 2018;193:71-9.

22. Devalla SK, et al. Glaucoma management in the era of artificial intelligence. British Journal of Ophthalmology. 2020;104(3):301-11.

23. Haleem MS, et al. A novel adaptive deformable model for automated optic disc and cup segmentation to aid glaucoma diagnosis. Journal of medical systems. 2018;42(1):20.

24. Omodaka K, et al. Classification of optic disc shape in glaucoma using machine learning based on quantified ocular parameters. PloS one. 2017;12(12):e0190012.

25. Noronha KP, et al. Automated classification of glaucoma stages using higher order cumulant features. Biomedical Signal Processing and Control. 2014;10:174-83.

26. Acharya UR, et al. A novel algorithm to detect glaucoma risk using texton and local configuration pattern features extracted from fundus images. Computers in biology and medicine. 2017:88:72-83.

27. Mookiah MRK, et al. Data mining technique for automated diagnosis of glaucoma using higher order spectra and wavelet energy features. Knowledge-Based Systems. 2012;33:73-82.

28. Yoo TK, Hong S. Artificial neural network approach for differentiating open-angle glaucoma from glaucoma suspect without a visual field test. Investigative Ophthalmology \& Visual Science. 2015;56(6):3957-66.

29. Li, F., et al., Deep learning-based automated detection of glaucomatous optic neuropathy on color fundus photographs. Graefe's Archive for Clinical and Experimental Ophthalmology, 2020: p. 1-17.

30. Kim SJ, Cho KJ, Oh S. Development of machine learning models for diagnosis of glaucoma. PLoS One. 2017;12(5):e0177726

31. Lee S-D, et al. Machine learning models based on the dimensionality reduction of standard automated perimetry data for glaucoma diagnosis. Artificial intelligence in medicine. 2019;94:110-6.

32. Chai Y, Liu H, Xu J. Glaucoma diagnosis based on both hidden features and domain knowledge through deep learning models. Knowledge-Based Systems. 2018;161:147-56.

33. Pathan S, et al. Automated segmentation and classification of retinal features for glaucoma diagnosis. Biomedical Signal Processing and Control. 2021;63:102244.

34. Fotouhi A, et al. Cohort profile: Shahroud eye cohort study. International journal of epidemiology. 2013;42(5):1300-8

35. Wirth, R. and J. Hipp. CRISP-DM: Towards a standard process model for data mining. in Proceedings of the 4th international conference on the practical applications of knowledge discovery and data mining. 2000. Springer-Verlag London, UK.

36. Han J, Kamber M, Pei J. Data mining concepts and techniques third edition. The Morgan Kaufmann Series in Data Management Systems. 2011;5(4):83-124.

37. Krawczyk B. Learning from imbalanced data: open challenges and future directions. Progress in Artificial Intelligence. 2016:5(4):221-32.

38. Breiman L. Bagging predictors. Machine learning. 1996;24(2):123-40.

39. Quinlan JR. Induction of decision trees. Machine learning. 1986;1(1):81-106.

40. Geurts P, Ernst D, Wehenkel L. Extremely randomized trees. Machine learning. 2006;63(1):3-42.

41. Breiman L. Random forests. Machine learning. 2001;45(1):5-32.

\section{Publisher's Note}

Springer Nature remains neutral with regard to jurisdictional claims in published maps and institutional affiliations. 